\title{
Tracking structural intermediates during Chikungunya virus membrane fusion using cryo-electron tomography and sub-tomogram averaging
}

Vidya Mangala Prasad ${ }^{1}$, Jelle Blijleven ${ }^{2}$, Jolanda Smit $^{2}$ and Kelly Lee ${ }^{3}$

${ }^{1}$ University of Washington, Seattle, Washington, United States, ${ }^{2}$ University of Groningen, Netherlands, United States, ${ }^{3}$ University of Washington, United States

Chikungunya virus (CHIKV) is a mosquito-borne alphavirus which has become a global concern with outbreaks in Asia, Africa, Europe and, recently, the Americas. It causes fever, myalgia and debilitating joint pain in infected patients, which in some cases can lead to severe arthritis or encephalitis ${ }^{1}$. CHIKV is an icosahedral virus which consists of 240 copies of E1-E2 surface glycoprotein heterodimers arranged on a lipid membrane envelope that surrounds an inner nucleocapsid core enclosing the viral RNA genome ${ }^{2}$ (Figure 1). Despite extensive structural and molecular studies on CHIKV and other alphaviruse ${ }^{3,4}$, there is still no direct, structural information on the conformational states that CHIKV undergoes during membrane fusion to release its genome into the host cell. We have used cryo-electron tomography (cryoET) and sub-tomogram averaging to tackle the challenge of trapping the dynamic process of CHIKV membrane fusion and visualizing its intermediate steps. By imaging CHIKV incubated with liposomes at varying $\mathrm{pH}$ and time points, we have pieced together a pathway for membrane fusion in CHIKV using direct experimental evidence. Multiple intermediate steps in the process including fusion loop attachment, formation of low $\mathrm{pH}$ induced E1 glycoprotein homotrimer, membrane mixing, hemifusion, fusion pore and release of the internal nucleocapsid can be clearly visualized (Figure 2). These results portray a complex interplay between the viral glycoproteins and membrane components, which varies starkly from existing fusion models that were primarily based on static high resolution structures of the virus and its glycoproteins. Sub-tomogram averaging efforts are currently underway to elucidate the 3D structures of various intermediates observed in the tomograms such as the glycoprotein organization surrounding the fusion pore, post-fusion glycoproteins and the released nucleocapsid. These structural analyses show the sequential changes that occur on CHIKV surface as it penetrates the target membrane, providing comprehensive, and in many cases unexpected, ultrastructural insights into the physical mechanisms by which the virus gains cellular entry. In addition, resolving the molecular process of CHIKV fusion provides a general platform to understand protein-mediated membrane fusion in other enveloped virus systems, cell-to-cell fusion, synaptic signaling and even placenta formation. 

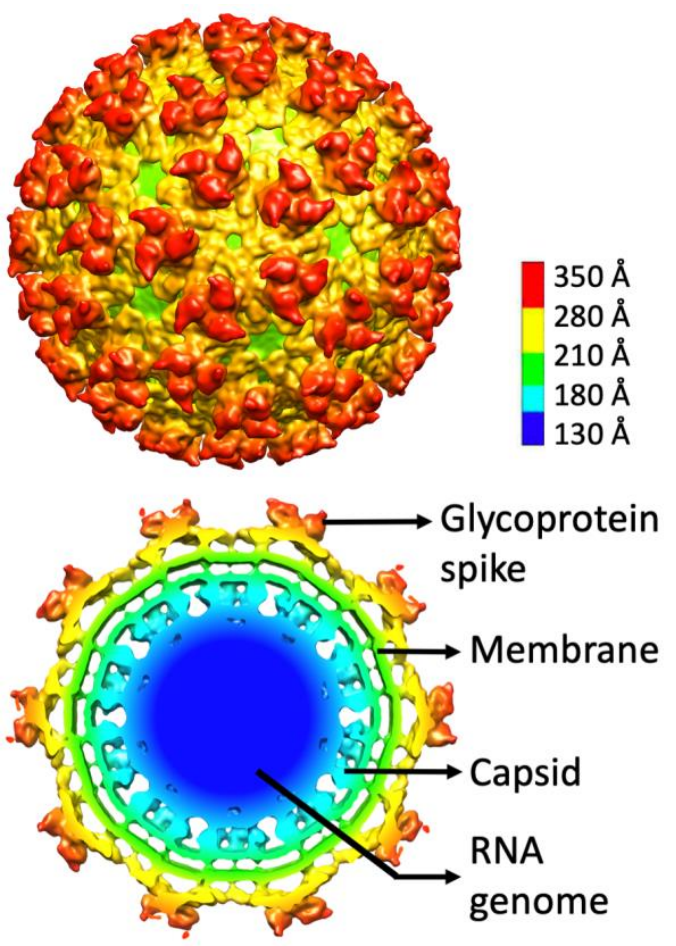

Figure 1. Cryo-EM structure of CHIKV sample used in the fusion studies. Resolution at 0.143 FSC is 6.5 $\AA$.

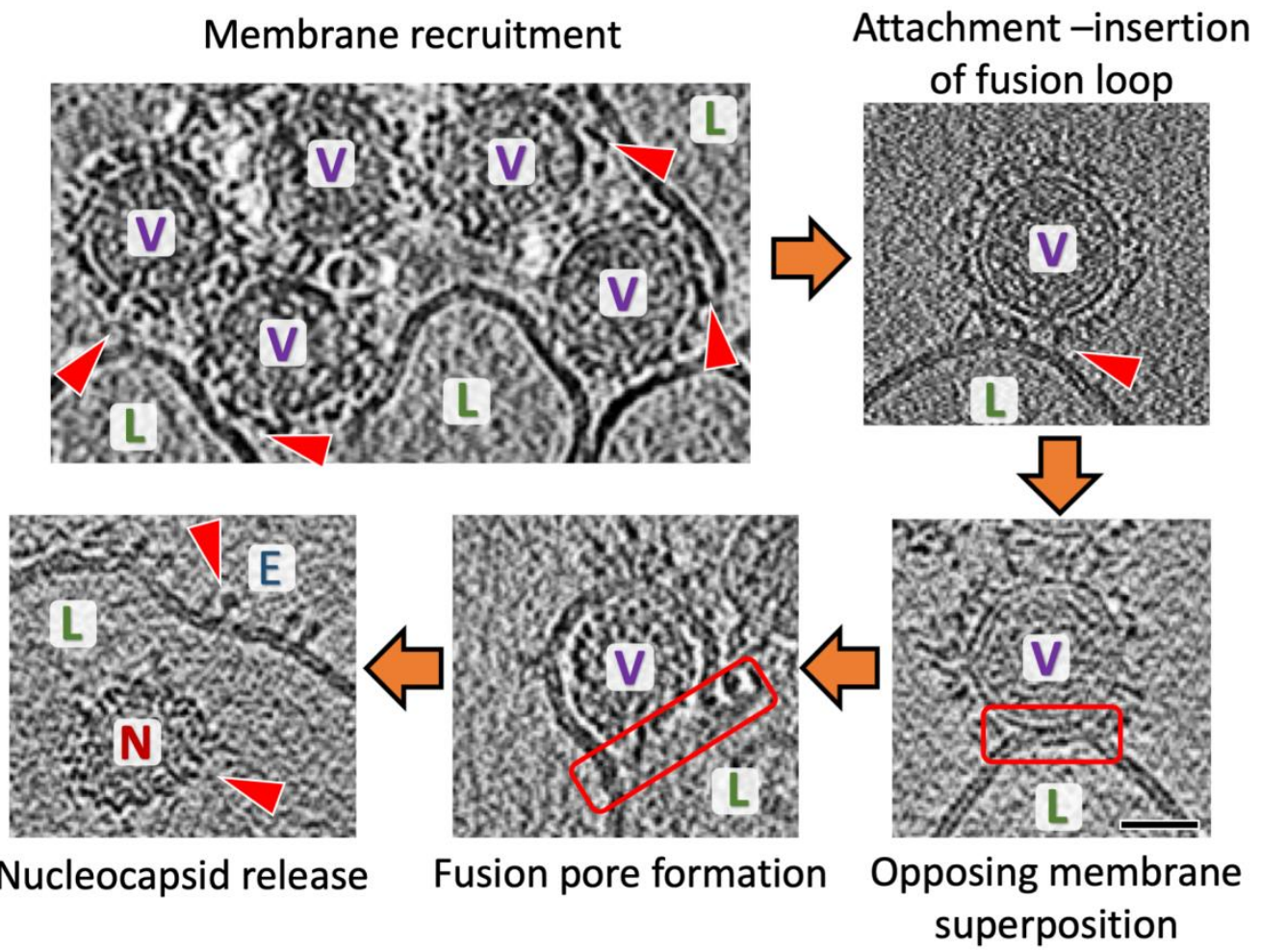

Figure 2. CHIKV membrane fusion imaged by Cryo-ET. The different intermediate states are labeled. Virions, liposomes and released nucleocapsid are indicated as $\mathrm{V}, \mathrm{L}$ and $\mathrm{N}$ respectively. External glycoproteins after fusion completion are labeled as E. Interaction interfaces are denoted by red arrows or red rectangles. Scale bar is $25 \mathrm{~nm}$. 


\section{References}

1. Weaver S.C and Lecuit M. 2015. Chikungunya Virus and the Global Spread of a Mosquito-Borne Disease. N Engl. J.Med. 372;13.

2. Sun S., Xiang Y., Akahata W., Holdaway H., Pal P., Zhang X., Diamond M.S., Nabel G.J., Rossmann M.G. 2013. eLife. 2:e00435.

3. Jose J., Snyder J.E., and Kuhn R.J. 2009. A structural and functional perspective of alphavirus replication and assembly. Future Micro. 4(7).

4. Holmes A.C., Basore K., Fremont D.H., and Diamond M.S. 2020. A Molecular Understanding of Alphavirus entry. Plos Path.16(10): e1008876. 